\title{
Upper Bound of Ergodic Capacity for MIMO Channels with Ricean-Fading using Majorization Theory
}

\author{
Antonio Alisson Pessoa Guimarães and Charles Casimiro Cavalcante
}

\begin{abstract}
This paper presents a closed-form upper bound for the ergodic capacity of spatially uncorrelated Ricean flat-fading channels with multiple-input multiple-output (MIMO) antennas. By considering an arbitrary number of antennas at both the transmitter and receiver sides and assuming that the channel state information (CSI) is completely unknown at the transmitter, the Majorization theory is used as a powerful mathematical tool, which allows us to derive an upper bound on the channel capacity for this class of channels. The upper bound obtained does not depend on the Ricean- $K$ factor. Moreover, in the high signal-to-noise-ratio (SNR) regimes, we give another approximation for Ricean-fading channel capacity. Finally, we present some numerical results that illustrate the validity of our approach.
\end{abstract}

Index Terms-Ergodic capacity, MIMO systems, Majorization theory, Ricean channel.

\section{INTRODUCTION}

$\mathbf{T}$ HE use of Multiple input multiple output (MIMO) antenna systems has attracted great interest, due to significant improvements in terms of spectral efficiency and reliability with respect to single input single output (SISO) antenna systems [1], [2]. In addition, one of the main focuses on MIMO systems is the study of the ergodic capacity, or Shannon capacity, which is viewed as an important performance measure [3].

This work investigates this performance measure on Ricean-fading channels. This statistical process is very useful to model the random signal fluctuations in various propagations environments and encompasses the Rayleigh-fading channels. Several researches, operating in Ricean-fading MIMO systems, have been published about closed-form upper bounds on the ergodic capacity. For example, in [4], an asymptotic upper bound was investigated on uncorrelated channels, while [5], [6] present bounds (upper and lower) for the correlated channel case. In turn, [7], [8] derived bounds and approaches for rank-1 MIMO channels.

Although the analysis on the ergodic capacity has been exhaustively analyzed in several settings [4]-[8], this subject still wakes up interest, especially when it is necessary to explore the limits of a given system. However, to obtain analytical closed-form formulas to ergodic capacity, in general, is a very difficult task. This complexity is due to derivation of the joint eigenvalue density distribution of the matrix

The authors are with the Wireless Telecommunications Research Group (GTEL), Department of Teleinformatics Engineering (DETI), Federal University of Ceará (UFC), Fortaleza-CE, Brazil, e-mails: \{alisson, charles\}@gtel.ufc.br, Phone/Fax: +55-85-33669470.
$\mathbf{H H}^{H}$, where $\mathbf{H}$ is defined as the channel matrix [7]-[10]. Most recently, by considering MIMO Nakagami- $m$ fading channels, Zhong et al. [11] have shown that it is possible to obtain capacity limits, without application of the joint eigenvalue distribution, by Majorization theory [12], [13]. This analysis has allowed them to specify a comparison between the diagonal elements of the positive semi-definite matrix $\mathbf{H H}^{H}$ and its singular values.

In this paper, we will focus on spatially uncorrelated Ricean-fading MIMO channels, where the matrix component of the channel, associated with the line-of-sight (LoS), is assumed to have an arbitrary rank. Specifically, using Majorization theory, we propose an ergodic capacity upper bound for this class of channels, which does not depend on the Ricean- $K$ factor. Further, from this result, we present a simple approximation for the ergodic capacity in high signal-to-noise ratio (SNR) regimes.

It is important to mention that this work differs from the current literature in MIMO systems, due to the mathematical treatment to the subject. In addition, we obtained a closed-form upper bound for the ergodic capacity without the use of the complex non-central Wishart distribution, and we consider that the matrix corresponding to the LoS has an arbitrary rank.

The remainder of this paper is organized as follows. In Section II, we describe the system model and the assumptions considered in this work. We list some results of Majorization theory that will be used in our study in Section III. Section IV provides an upper bound and an approximation in high SNR regimes for the ergodic capacity over Ricean-fading MIMO channels. Simulation results are presented in Section V. We conclude this paper in Section VI.

Throughout this paper, matrices and vectors will be represented by bold uppercase and lowercase letters, respectively. We use $\mathbf{I}$ or $\mathbf{I}_{p}$ for the identity matrix of dimension $p \times p$. The operators $(\cdot)^{H}, \otimes$ and $\prec$ denote the conjugate transpose, Kronecker product and majorization relation, respectively. In turn, $\operatorname{det}(\cdot)$ denotes the determinant of a matrix and $\mathbb{E}\{\cdot\}$ represents the expected value operator. Finally, the result of the operators $\mathrm{d}(\cdot)$ and $\lambda(\cdot)$ are vectors and denote the diagonal elements and the eigenvalues, respectively, of a given Hermitian matrix.

\section{SySTEM MODEL}

Let us consider a single-user MIMO communication system with $n_{T}$ transmit antennas and $n_{R}$ receive antennas over a Ricean flat-fading channel. In addition, we have assumed that 
the channel state information (CSI) is completely unknown at the transmitter.

The received signal vector $\mathbf{y} \in \mathbb{C}^{n_{R} \times 1}$ is given by

$$
\mathbf{y}=\mathbf{H x}+\mathbf{n},
$$

where the data vector $\mathbf{x} \in \mathbb{C}^{n_{T} \times 1}$ is the transmitted signal vector satisfying the total power constraint $\mathbb{E}\left\{\|\mathbf{x}\|^{2}\right\} \leq P_{T}$. The noise vector $\mathbf{n} \in \mathbb{C}^{n_{R} \times 1}$ is considered to be Zero-Mean Circular Symmetric Complex Gaussian (ZMCSCG) with covariance matrix $N_{0} \mathbf{I}_{n_{R}}$, i.e., $\mathbf{n} \sim \mathcal{C N}\left(\mathbf{0}, N_{0} \mathbf{I}_{n_{R}}\right)$. The elements of the channel matrix $\mathbf{H}=\left[h_{i j}\right] \in \mathbb{C}^{n_{R} \times n_{T}}$ are assumed to have the same mean square value, and equal to $\Omega$, that is, $\mathbb{E}\left\{\left|h_{i j}\right|^{2}\right\}=\Omega$. In this paper, we have considered that the envelope $\left|h_{i j}\right|$ of the complex entries $h_{i j}$ follows a Rice distribution given by [14]:

$p_{\left|h_{i j}\right|}(h)=\frac{2(K+1)}{\Omega} h \exp \left(-\frac{(K+1) h^{2}}{\Omega}-K\right) I_{0}\left(2 h \sqrt{\frac{K(K+1)}{\Omega}}\right)$,

where the real number $K$ is the Ricean- $K$ factor and represents the ratio of deterministic energy to scattered energy. The term $I(\cdot)$ is the Bessel function of the first kind. Furthermore, we have considered that the channel is spatially uncorrelated, thus the matrix $\mathbf{H}$ can be represented by the following model [8], [15]:

$$
\mathbf{H}=\sqrt{\frac{\Omega K}{K+1}} \overline{\mathbf{H}}+\sqrt{\frac{\Omega}{K+1}} \mathbf{H}_{w},
$$

where $\overline{\mathbf{H}}$ is a deterministic (specular) matrix, with an arbitrary rank $L$, which corresponds to the LoS. The term $\mathbf{H}_{w}=\left[\widetilde{h}_{i j}\right]$ is a random (scattered) matrix whose elements are independent and identically distributed (i.i.d.), ZMCSCG and unit variance, i.e., $\mathbf{H}_{w} \sim \mathcal{C N}\left(\mathbf{0}, \mathbf{I}_{n_{R}} \otimes \mathbf{I}_{n_{T}}\right)$. It is important to mention that, if $K=0$ then we have an uncorrelated Rayleigh flat-fading channel, while $K \rightarrow \infty$ corresponds to non-fading channels [14], [16].

Under the assumptions described above, the ergodic capacity is given by [7], [15], [17], [18]

$$
\bar{C}=\mathbb{E}\left\{\log _{2} \operatorname{det}\left(\mathbf{I}+\frac{\rho}{n_{T}} \boldsymbol{\Gamma}\right)\right\},
$$

where $\rho \triangleq \frac{P_{T}}{N_{0}}$ is the average SNR at the receiver and

$$
\boldsymbol{\Gamma}= \begin{cases}\mathbf{H H}^{H}, & \text { if } n_{R} \leq n_{T} \\ \mathbf{H}^{H} \mathbf{H}, & \text { if } n_{R}>n_{T} .\end{cases}
$$

Next, let us define the terms $m=\min \left\{n_{R}, n_{T}\right\}$ and $n=\max \left\{n_{R}, n_{T}\right\}$. Then, $\boldsymbol{\Gamma}$ is always a square matrix of order $m \times m$.

Finally, the channel matrix $\mathbf{H}$ has a matrix-variate complex Gaussian distribution, i.e., [7], [19]

$$
\mathbf{H} \sim \mathcal{C N}\left(\sqrt{\frac{\Omega K}{K+1}} \overline{\mathbf{H}}, \frac{\Omega}{K+1} \mathbf{I}_{n_{R}} \otimes \mathbf{I}_{n_{T}}\right) .
$$

Throughout the paper, we assume that the number of receive antennas does not exceed the number of transmit antennas, i.e., $n_{R} \leq n_{T}$. In addition, using the identity $\operatorname{det}(\mathbf{I}+\mathbf{A B})=\operatorname{det}(\mathbf{I}+\mathbf{B A})$, all results can be extended to the case $n_{R}>n_{T}$.

\section{MAJORIZATION THEORY}

For the development proposed in this work, some basic results and definitions of Majorization theory will be necessary. The mathematical fundamentals related to the Majorization Theory can be found in the classical reference, [12] and some problems in wireless communications are elegantly studied in [20], [21] using this theory. Moreover, this tool also helps in solving some optimization problems [21]-[23]. Here, we present the most relevant elements for our purposes.

Definition 1: $\left[12,1\right.$. A.1] Let $\mathbf{x}=\left(x_{1}, x_{2}, \cdots, x_{n}\right)$ and $\mathbf{y}=\left(y_{1}, y_{2}, \cdots, y_{n}\right)$ be vectors in $\mathbb{R}^{n}$. We denote by $[\mathbf{x}]=\left(x_{[1]}, x_{[2]}, \cdots, x_{[n]}\right)$ the vector obtained by re-ordering the coordinates of $\mathbf{x}$ in a decreasing order, that is, $x_{[1]} \geq x_{[2]} \geq$ $\cdots \geq x_{[n]}$. The vector $\mathbf{x}$ is said majorized by $\mathbf{y}$ (represented by $\mathbf{x} \prec \mathbf{y}$ ), if

$$
\begin{aligned}
& \sum_{i=1}^{k} x_{[i]} \leq \sum_{i=1}^{k} y_{[i]}, \quad 1 \leq k \leq n-1, \\
& \sum_{i=1}^{n} x_{[i]}=\sum_{i=1}^{n} y_{[i]} .
\end{aligned}
$$

Definition 2: [12, 3.A.1] A real-valued function $\phi(\cdot)$ on $\mathbb{R}^{n}$ is called Schur-convex if

$$
\mathbf{x} \prec \mathbf{y} \Rightarrow \phi(\mathbf{x}) \leq \phi(\mathbf{y}) .
$$

Similarly, $\phi(\cdot)$ is said to be Schur-concave if

$$
\mathbf{x} \prec \mathbf{y} \Rightarrow \phi(\mathbf{x}) \geq \phi(\mathbf{y}) .
$$

Lemma 1: [12, 3.C.1] Consider the real-valued function $\phi(\cdot)$ on $\mathbb{R}^{n}$. If $g: \mathbb{R} \rightarrow \mathbb{R}$ is concave, then $\phi(\cdot)$, defined by

$$
\phi(\mathbf{x})=\sum_{i=1}^{n} g\left(x_{i}\right)
$$

is Schur-concave.

Lemma 2: $[12,9 . \mathrm{B} .1]$ If $\mathbf{A} \in \mathbb{C}^{n \times n}$ is a Hermitian matrix, then

$$
\mathrm{d}(\mathbf{A}) \prec \lambda(\mathbf{A}) .
$$

\section{CAPACITy UPPER Bound OF MIMO UNDER RICEAN-FADING}

In this paper, we present a different approach for obtaining upper bounds on the ergodic capacity of uncorrelated Ricean MIMO flat-fading channels. This theory avoids the use of the complex non-central Wishart distribution [5], [7]. Specifically, we derive the upper bound on Schur-concave function in terms of the eigenvalues and diagonal elements of a given Hermitian matrix, as shown in the next theorem.

Theorem 1: The ergodic capacity of MIMO Ricean-fading channel is upper bounded by

$$
\bar{C} \leq C_{\text {up }}=m \log _{2}(1+\rho \Omega) .
$$

Proof: Firstly, we will transform the channel matrix in order to simplify our calculus. Specifically, we define

$$
\mathbf{W}=\sqrt{\frac{1+K}{\Omega}} \mathbf{H} .
$$


Thus, the elements of $\mathbf{W}=\left[w_{i j}\right] \in \mathbb{C}^{n_{R} \times n_{T}}$ have constant power, i.e., $\mathbb{E}\left[\left|w_{i j}\right|^{2}\right]=K+1$, and this matrix has the following decomposition

$$
\mathbf{W}=\sqrt{K} \overline{\mathbf{H}}+\mathbf{H}_{w} .
$$

Furthermore, $\mathbf{W}$ has a matrix-variate complex Gaussian distribution [19]

$$
\mathbf{W} \sim \mathcal{C N}\left(\sqrt{K} \overline{\mathbf{H}}, \mathbf{I}_{m} \otimes \mathbf{I}_{n}\right) .
$$

In words, the new channel matrix $\mathbf{W}$ has a matrix-mean $\sqrt{K} \overline{\mathbf{H}}$ and covariance-matrix equal to $\mathbf{I}_{m \times n}$.

On the other hand, from Equation (13), the ergodic capacity in Equation (4) is written as

$$
\bar{C}=\mathbb{E}\left\{\log _{2} \operatorname{det}\left(\mathbf{I}_{m}+\frac{\rho \Omega}{n(K+1)} \mathbf{W} \mathbf{W}^{H}\right)\right\} .
$$

In turn, let us consider the vectors

$$
\lambda\left(\mathbf{W} \mathbf{W}^{H}\right)=\left(\lambda_{1}, \lambda_{2}, \cdots, \lambda_{m}\right)
$$

and

$$
\mathrm{d}\left(\mathbf{W} \mathbf{W}^{H}\right)=\left(d_{1}, d_{2}, \cdots, d_{m}\right),
$$

which correspond to the eigenvalues and diagonal elements of the Hermitian matrix $\mathbf{W} \mathbf{W}^{H}$, respectively. Under these assumptions, the ergodic capacity in (16) can be written as [15]

$$
\bar{C}=\mathbb{E}\left\{\sum_{i=1}^{m} \log _{2}\left(1+\frac{\rho \Omega}{n(K+1)} \lambda_{i}\right)\right\} .
$$

Additionally, based on Lemma 2, we have

$$
\mathrm{d}\left(\mathbf{W} \mathbf{W}^{H}\right) \prec \lambda\left(\mathbf{W} \mathbf{W}^{H}\right) .
$$

Now, let the function $\phi: \mathbb{R}^{p} \rightarrow \mathbb{R}$ be defined by

$$
\phi(\mathbf{x})=\sum_{i=1}^{p} \log _{2}\left(1+\frac{\rho \Omega}{n(K+1)} x_{i}\right),
$$

and the real-valued concave function $g(x)=\log _{2}(1+k x)$, with $k>0$. Thus, based on Lemma 1 , we guarantee that the function $\phi(\cdot)$ in Equation (21) is Schur-concave and

$$
\phi\left(\mathrm{d}\left(\mathbf{W} \mathbf{W}^{H}\right)\right) \geq \phi\left(\lambda\left(\mathbf{W} \mathbf{W}^{H}\right)\right) .
$$

Applying the expectation operator $\mathbb{E}\{\cdot\}$ in Equation (22), we obtain

$$
\bar{C}=\mathbb{E}\left\{\phi\left(\lambda\left(\mathbf{W} \mathbf{W}^{H}\right)\right)\right\} \leq \mathbb{E}\left\{\phi\left(\mathrm{d}\left(\mathbf{W} \mathbf{W}^{H}\right)\right)\right\} .
$$

Therefore,

$$
\begin{aligned}
\bar{C} & \leq \mathbb{E}\left\{\sum_{i=1}^{m} \log _{2}\left(1+\frac{\rho \Omega}{n(K+1)} d_{i}\right)\right\} \\
& =\mathbb{E}\left\{\log _{2}\left(\prod_{i=1}^{m}\left(1+\frac{\rho \Omega}{n(K+1)} d_{i}\right)\right)\right\} .
\end{aligned}
$$

According to Jensen's inequality [12, 16.C.1], we have

$$
\bar{C} \leq \log _{2}\left(\mathbb{E}\left\{\prod_{i=1}^{m}\left(1+\frac{\rho \Omega}{n(K+1)} d_{i}\right)\right\}\right) .
$$

Note that $d_{i}=\sum_{j=1}^{n}\left|w_{i j}\right|^{2}$, for $i=1,2, \cdots, m$, and $\mathbb{E}\left\{d_{i}\right\}=n(K+1)$. Hence, the mean of the products of $d_{1}, d_{2}, \cdots, d_{m}$ is given by

$$
\begin{aligned}
\mathbb{E}\left\{\prod_{i=1}^{m} d_{i}\right\} & =\sum_{j_{1}=1}^{n} \sum_{j_{2}=1}^{n} \cdots \sum_{j_{m}=1}^{n} \mathbb{E}\left\{\prod_{k=1}^{m}\left|w_{k j_{k}}\right|^{2}\right\} \\
& =(n \cdot(K+1))^{m} .
\end{aligned}
$$

In summary, with the result from (26), the mean value term in Equation (25) is given by

$$
\mathbb{E}\left\{\prod_{i=1}^{m}\left(1+\frac{\rho \Omega}{n(K+1)} d_{i}\right)\right\}=(1+\rho \Omega)^{m} .
$$

Hence, the ergodic capacity of MIMO Ricean-fading channel is upper bounded by

$$
\bar{C} \leq C_{\text {up }}=m \log _{2}(1+\rho \Omega) .
$$

This completes the proof of the theorem.

Observe that the upper bound obtained in (28) depends on the numbers of antennas, the SNR value and the power $\Omega$, in each entry of the MIMO channel matrix. Moreover, our result is independent of the Ricean- $K$ factor and the Ricean and Rayleigh fading channels have the same upper bound when we consider the same numbers of antennas.

In Section V, the upper bound given by Equation (28) is compared with a similar result presented by [8, Theorem 4]

$$
C_{U_{1}}=\log _{2}\left(\sum_{i=0}^{m}\left(\begin{array}{c}
m \\
i
\end{array}\right)\left(\frac{\rho}{n}\right)^{i} \frac{n !}{(n-i) !} \frac{1+i K}{(K+1)^{i}}\right),
$$

when the LoS matrix component has a unit-rank and $\Omega=1$.

Finally, at high value of SNR (large $\rho$ ), the function $m \log _{2}(1+\rho \Omega)$ can be approximated by $m \log _{2}(\rho \Omega)$ [15].

\section{Numerical Results}

In this section, we compare our upper bound with the one proposed in [8], for uncorrelated Ricean flat-fading channels, with $K=1$ and $K=3$. Here, we consider $\Omega=1$, a unit-rank for the LoS matrix component, and the number of receive antennas does not exceed the number of transmit antennas.

Figures 1 and 2 depict the closed-form upper bounds given in [8] and the one proposed in this work, together with simulation results, for $K=1$ and $K=3$, respectively. Figure 3 shows the same upper bounds, but using the high SNR regimes approximation.

The simulation results in Figure 1 and 2 illustrate that, for the SISO case, the upper bound proposed in this work and the one suggested (see Equation (29)) by [8] are identical. For the MIMO case, we observe the upper bound given in [8] is a little tighter than our upper bound, and the difference is more evident when the value of Ricean $K$-factor is increased. We have the same behavior for high SNR values (see Figure 3). The reason for this difference is that our result is independent of the Ricean $K$-factor. Thus, we fail to capture the impact of the line-of-sight components.

However, the upper bound $C_{\text {up }}$ given in Equation (28), obtained using Majorization theory, has the advantages of 
being a simple analytical closed-form formula, an easy computational treatment and, more importantly, the method employed is less complex than traditional methods, which use the complex non-central Wishart distribution [5], [7]. Additionally, we highlight that this method can be useful to obtain upper bounds on other types of fading channels.

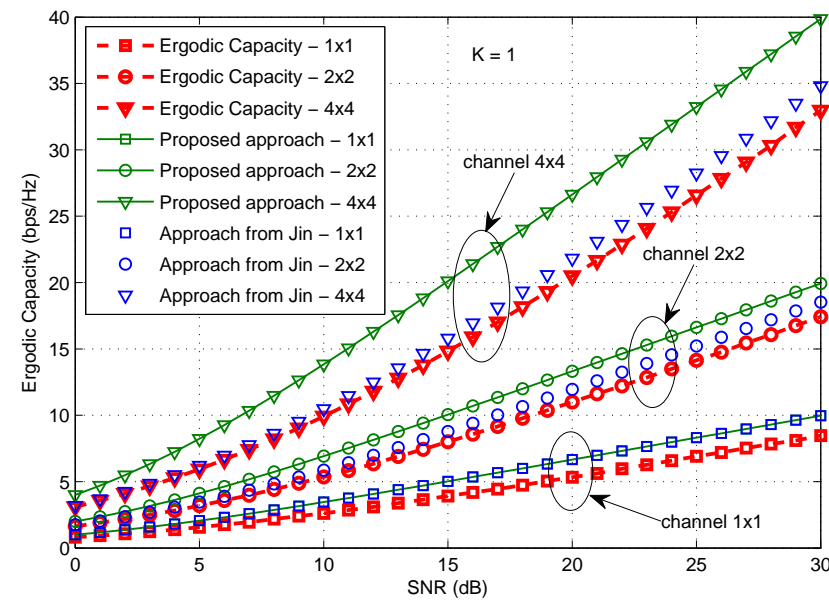

Fig. 1. Comparison between the upper bound proposed by this paper and the obtained by [8] for $1 \times 1,2 \times 2$ and $4 \times 4$ on Ricean-fading MIMO channels with $K=1$.

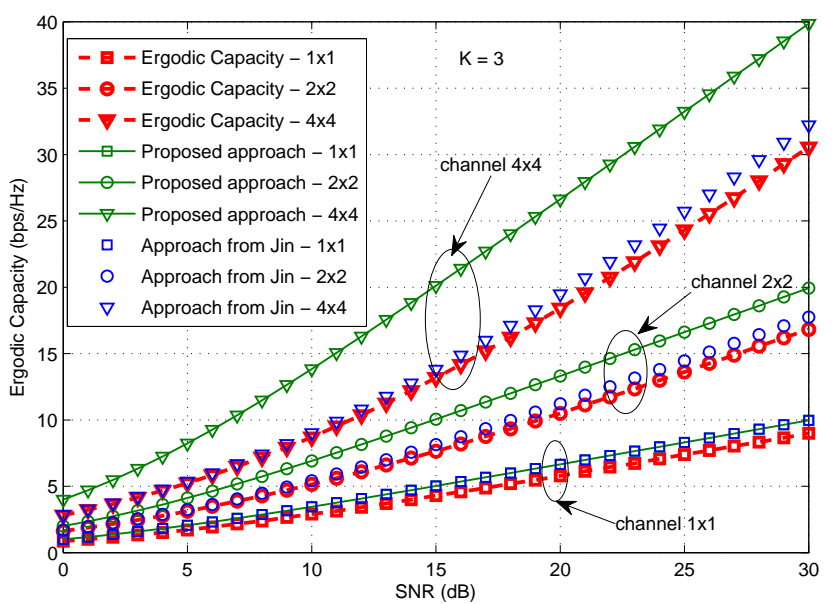

Fig. 2. Comparison between the upper bound proposed by this paper and the obtained by [8] for $1 \times 1,2 \times 2$ and $4 \times 4$ on Ricean-fading MIMO channels with $K=3$.

\section{CONCLUSION}

In this work, we have investigated the ergodic capacity of a point-to-point MIMO system under a spatially uncorrelated Ricean flat-fading channel when the channel state information is completely unknown at the transmitter. We have considered that the LoS matrix component has an arbitrary rank. Using Majorization theory, we have obtained a closed-form upper bound for the capacity ergodic in a simpler way than previous works. In addition, we have concluded that our result is independent of the Ricean- $K$ factor. In the high SNR regimes,

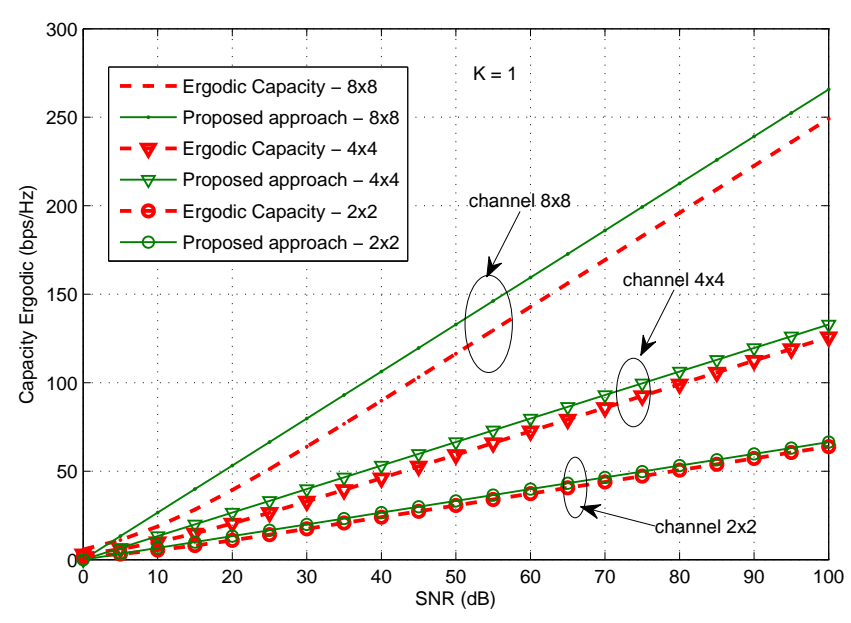

Fig. 3. Approaches to the ergodic capacity in the cases $2 \times 2,4 \times 4$ and $8 \times 8$ for high SNR regimes, with $K=1$.

we have obtained an approximation to ergodic capacity by considering the upper bound obtained. As far as the contributions of the paper are concerned, we have presented a simple closed-form upper bound on ergodic capacity when the LoS matrix component has an arbitrary rank, as well as an approximation for high SNR regimes on Ricean-fading without the Ricean- $K$ factor. A perspective for future works is to evaluate upper and lower bounds and approximations in high e low SNR regimes for the ergodic capacity on other fading distributions with the use of the majorization theory on MIMO and broadcast channels.

\section{ACKNOWLEDGMENTS}

The authors would like to thank CAPES, CNPq (Grant No. 30677/2011-9) and PRONEX/FUNCAP for the partial financial support. They would also like to thank the Editor and the anonymous reviewers for their helpful comments that considerably improved the quality of this paper.

\section{REFERENCES}

[1] I. E. Telatar, "Capacity of multi-antenna Gaussian channels," Europ. Trans. Telecommun., vol. 10, no. 6, pp. 585-595, nov. 1999.

[2] G. J. Foschini and M. J. Gans, "On limits of wireless communications in a fading environment when using multiple antennas," Wireless Pers. Comm., vol. 6, pp. 311-335, 1998.

[3] T.M. Cover and J.A. Thomas, Elements of Information Theory, Wiley Series in Telecommunications and Signal Processing. Wiley-Interscience, 2006.

[4] G. Lebrun, M. Faulkner, M. Shafi, and P. J. Smith, "MIMO Ricean Channel Capacity: An Asymptotic Analysis," IEEE Trans. on Wireless Comm., vol. 5, no. 6, pp. 1343-1350, June 2006.

[5] M. R. McKay and I. B. Collings, "General Capacity Bounds for Spatially Correlated Rician MIMO Channels," IEEE Trans. on Inf. Theory, vol. 51, no. 9, pp. 3121-3145, Sept. 2005.

[6] B. O. Hogstad, G. Rafiq, V. Kontorovitch, and M. Patzold, "Capacity studies of spatially correlated MIMO Rice channels," in Wireless Pervasive Computing (ISWPC), 2010 5th IEEE International Symposium on, May 2010, pp. 45-50.

[7] S. K. Jayaweera and H. V. Poor, "On the Capacity of Multiple Antenna Systems in Rician Fading," IEEE Trans. on Comm., vol. 4, no. 3, pp. $1102-1111,2005$.

[8] S. Jin, X. Gao, and X. You, "On the Ergodic Capacity of Rank-1 Ricean-Fading MIMO Channels," IEEE Trans. on Inf. Theory, vol. 53 , no. 2, pp. 502-517, Feb. 2007. 
[9] G. Fraidenraich, O. Lévêque, and J. M. Cioffi, "On the MIMO Channel Capacity for the Nakagami- $m$ Channel," IEEE Trans. on Inf. Theory, vol. 54, no. 8, pp. 3752-3757, Aug. 2008.

[10] G. Alfano, A. Lozano, A. M. Tulino, and S. Verdú, "Mutual information and eigenvalue distribution of MIMO Ricean channels," in IEEE Int. Symp. Information Theory \& Applications (ISITA'04), Oct. 2004.

[11] C. Zhong, K.-K. Wong, and S. Jin, "On the Ergodic Capacity of MIMO Nakagami-Fading Channels," IEEE Trans. on Signal Proc., vol. 57, no. 9, pp. 3613-3623, Sept. 2009.

[12] A. W. Marshall and I. Olkin, Theory of Majorization and Its Applications, Academic Press, 1979.

[13] R. Bhatia, Matrix Analysis, Springer, 1997.

[14] A. Goldsmith, Wireless Communications, Cambridge University Press, 2005.

[15] A. Paulraj, R. Nabar, and D. Gore, Introduction to Space-Time Wireless Communications, Cambridge University Press, 2003.

[16] D. Tse and P. Viswanath, Fundamentals of Wireless Communication, Cambridge University Press, 2009.

[17] E. Biglieri and G. Taricco, "Transmission and reception with multiple antennas: theoretical foundations," Found. Trends Commun. Inf. Theory, vol. 1, pp. 183-332, 2004.

[18] E. Biglieri, R. Calderbank, A. Constantinides, A. Goldsmith, A. Paulraj, and H. V. Poor, MIMO Wireless Communications, Cambridge University Press, New York, NY, USA, 2010.

[19] X. Zhang, D.P. Palomar, and B. Ottersten, "Statistically Robust Design of Linear MIMO Transceivers," Signal Processing, IEEE Transactions on, vol. 56, no. 8, pp. 3678-3689, 2008.

[20] E. Jorswieck and $\mathrm{H}$. Boche, Majorization and Matrix-Monotone Functions in Wireless Communications, vol. 3, Found. Trends Commun. Inf. Theory, 2007.

[21] D. P. Palomar and Y. Jiang, "MIMO Transceiver Design via Majorization Theory," Found. Trends Commun. Inf. Theory, vol. 3, no. 4-5, pp. 331-551, 2006.

[22] D. P. Palomar, J. M. Cioffi, and M. A. Lagunas, "Uniform Power Allocation in MIMO Channels: A Game-Theoretic Approach," IEEE Transactions on Information Theory, vol. 49, no. 7, pp. 1707-1727, 2003.

[23] A. A. P. Guimarães and I. M. Guerreiro and L. M. C. Sousa and T. F. Maciel and C. C. Cavalcante, "A (Very) Brief Survey on Optimization Methods for Wireless Communications Systems," in 7th International Telecommunications Symposium (ITS 2010), Manaus, AM, Sep. 2010,
Proceedings of the 7th International Telecommunications Symposium (ITS 2010).

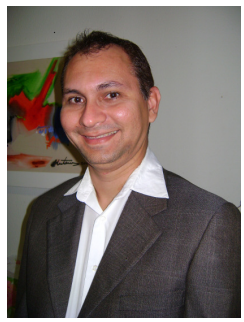

Antonio Alisson Pessoa Guimarães received the Bachelor's degree in Mathematics and the Master of Science degree in Teleinformatics Engineering from the Federal University of Ceará (UFC), Brazil, in 2004 and 2010, respectively. He was substitute professor of the Mathematics Department (UFC) from 2006 to 2008. Nowadays, he is $\mathrm{Ph} . \mathrm{D}$. student at the Teleinformatics Engineering Department and a member of the Research Group in Telecommunications Wireless (GTEL). His research interests include Adaptive Filtering, Convex Optimization theory, Majorization theory, Information Geometry and performance limits of wireless MIMO systems from the Information-theoretic.

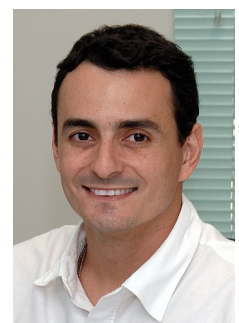

Charles Casimiro Cavalcante received the B.Sc and M.Sc in Electrical Engineering from the Federal University of Ceará (UFC), Brazil, in 1999 and 2001, respectively, and the D.Sc. degree from the University of Campinas (UNICAMP), Brazil, in 2004. He has held a grant for Scientific and Technological Development from 2004 to 2007 and since March 2009 he has a grant of Scientific Research Productivity both from the Brazilian Research Council (CNPq). From March 2007 to November 2008 he was a visiting professor at Teleinformatics Engineering Department of UFC and since November he is an Assistant Professor at the same department and university holding the Statistical Signal Processing chair. He has been working on signal processing strategies for communications where he has several papers published in journal and conferences, has authored two international patents and he has worked on several funded research projects on the wireless communication area. He is also a co-author of the book Unsupervised Signal Processing: Channel Equalization and Source Separation, published by CRC Press. He is a researcher of the Wireless Telecommunications Research Group (GTEL) where he leads research on signal processing and wireless communications. His main research interests are in signal processing for communications, blind source separation, wireless communications, and statistical signal processing. 